\title{
Using Experimental Models to Identify Pathogenic Pathways and Putative Disease Management Targets in Bronchopulmonary Dysplasia
}

\author{
Rory E. Morty \\ Department of Lung Development and Remodelling, Max Planck Institute for Heart and Lung Research, Bad Nauheim, \\ Germany; Department of Internal Medicine (Pulmonology), University of Giessen and Marburg Lung Center (UGMLC), \\ member of the German Center for Lung Research (DZL), Giessen, Germany
}

\section{Keywords}

Hyperoxia - Animal models - Bronchopulmonary dysplasia

\section{Abstract}

Bronchopulmonary dysplasia (BPD) is a common and serious complication of preterm birth. Limited pharmacological and other medical interventions are currently available for the management of severely affected, very preterm infants. BPD can be modelled in preclinical studies using experimental animals, and experimental animal models have been extremely valuable in the development of hallmark clinical management strategies for BPD, including pulmonary surfactant replacement and single-course antenatal corticosteroids. A gradual move away from large animal models of BPD in favor of term-born rodents has facilitated the identification of a multitude of new mechanisms of normal and stunted lung development, but this has also potentially limited the utility of experimental animal models for the identification of pathogenic pathways and putative disease management targets in BPD. Indeed, more recent pharmacological interventions for the management of BPD that have been

karger@karger.com www.karger.com/neo Karger $\stackrel{\text { ' }}{=}$
(C) 2020 The Author(s) Parger AG, Basel access

This is an Open Access article licensed under the Creative Commons Attribution-NonCommercial-4.0 International License (CC BY-NC) (http://www.karger.com/Services/OpenAccessLicense), applicable to the online version of the article only. Usage and distribution for commercial purposes requires written permission. validated in randomized controlled trials have relied very little on preclinical data generated in experimental animal models. While rodent-based models of BPD have tremendous advantages in terms of the availability of genetic tools, they also have considerable drawbacks, including limited utility for studying breathing mechanics, gas exchange, and pulmonary hemodynamics; and they have a less relevant clinical context where lung prematurity and a background of infection are now rarely present in the pathophysiology under study. There is a pressing need to refine existing models to better recapitulate pathological processes at play in affected infants, in order to better evaluate new candidate pharmacological and other interventions for the management of BPD.

(c) 2020 The Author(s)

Published by S. Karger AG, Basel

Preterm-born infants with respiratory failure are at risk for the development of bronchopulmonary dysplasia (BPD), first described in 1967 [1]. In affected infants, the immature, developing lung is exposed to a series of injurious stimuli (oxygen injury, mechanical trauma, infection 
and inflammation, amongst others) culminating in a syndrome where lung developmental programs are deregulated, creating disturbances to the delicate lung architecture, which are further distorted by tissue repair programs that are engaged in response to injury [2]. In addition to presenting a clinical challenge in a neonatal intensive care setting, BPD survivors are at risk for compromised respiratory function and adverse neurodevelopmental effects which persist into adolescence and adulthood [2]. Advances in the medical management of preterm birth have caused the spectrum of affected infants to shift over time, with "less preterm" infants no longer affected, while extremely preterm infants now survive, but develop severe disease [3]. This shift in the affected infant population was accompanied by evolving BPD pathology which has shifted from that first described in 1967 [1] to what is now largely a stunting of distal lung development [4].

The first description of BPD in 1967 [1] heralded the advent of a new era in neonatology and pediatric pulmonology, and inspired tremendous interest in understanding lung development, particularly the development of the alveoli and their associated vasculature [5]. Furthermore, there was much enthusiasm for the generation of appropriate animal models to study pathological processes at play in infants with BPD, with the aim to identify putative disease-driving pathways that could be therapeutically targeted. To this end, shortly after the first description of BPD in 1967, the same groups of investigators presented radiological and histological evidence of pulmonary oxygen toxicity in two new animal models: newborn guinea pigs in 1969 [6] and newborn mice in 1975 [7]. Since then, a broad spectrum of animal models of BPD has been developed, including term-born mice and rats, and preterm-born rabbits, pigs, sheep and baboons [reviewed in 8,9]. The engagement of experimental animals in BPD research proved pivotal for the development of the first two key pharmacological interventions that had a tremendous impact on the medical management of preterm-born infants: surfactant therapy $[10,11]$ and single-course antenatal corticosteroids [12].

Following the discovery by Mary Ellen Avery and Jere Mead in 1959 that extracts from the lungs of preterm infants with respiratory distress syndrome effectively lacked surfactant [13], three studies undertaken on preterm rabbits and preterm primates between 1972 and 1978 revealed that intratracheal application of pulmonary surfactant improved lung expansion and ventilation in preterm experimental animals [reviewed in 10]. This paved the way for the first successful administration of pulmo- nary surfactant to manage preterm birth in humans [14]. For antenatal steroids, the discovery was more serendipitous and followed studies on premature parturition by Liggins [15]. While studying the effects of dexamethasone on parturition in fetal sheep in 1969, Liggins observed that while the steroid caused premature birth, the lambs had partial aeration of their lungs unexpected at these gestations. He believed that corticosteroids acted to trigger the appearance of pulmonary surfactant [15]. Liggins, an obstetrician, then conducted with Howie, a pediatrician, the first clinical trial in 1972 that demonstrated the utility of corticosteroids to reduce the incidence of respiratory distress syndrome in the newborn after preterm labor [16]. Other preclinical studies included the observation in 1972, also by Mary Ellen Avery, that lung maturation was accelerated and survival increased in preterm-delivered rabbits that had received hydrocortisone in utero [17]. Furthermore, prenatal administration of dexamethasone to pregnant rats protected offspring against the deleterious effects of oxygen toxicity and promoted postnatal lung growth [18]. Thus, experimental animal models proved essential for the development of the two most important pharmacological interventions employed to manage preterm birth. In contrast, more recent pharmacological interventions that have demonstrable utility in the clinical management of preterm neonates with, or at risk for, development of BPD appear to have benefited far less from preclinical studies in experimental animal models.

A recent systematic review of 47 randomized controlled trials (RCTs) conducted between 1992 and 2014 that assessed the utility of pharmacological interventions for the prevention of BPD [19] revealed that of 21 pharmacological interventions, only 5 (tested in 13 RCTs with 4,794 enrolled patients) reduced the incidence of BPD: vitamin A, caffeine citrate, postnatal dexamethasone, inositol and clarithromycin. Interestingly, preclinical studies in experimental animal models of BPD provided very little support to underpin the clinical trials establishing the success of these 5 pharmacological interventions.

Intramuscular vitamin A (retinol and its derivatives) for prevention of BPD has been supported by a 1992 RCT which was confirmed by a 2011 meta-analysis [discussed in 19]. However, these studies were driven by observations that preterm infants were deficient in vitamin $\mathrm{A}$ at birth, and that preterm neonates who developed BPD had protracted periods of suboptimal plasma vitamin A levels during the early postnatal period. Although dynamic changes in expression of retinoic acid receptors over the course of postnatal lung development in experimental 
animals were noted as early as 1976 [20], interventional preclinical studies exploring the use of vitamin A and its derivatives in experimental animal models of BPD took place in parallel with the RCT. These preclinical studies, undertaken between 2000 and 2002, were largely negative, and included the demonstration that antenatal retinoic acid (the physiologically active vitamin $\mathrm{A}_{1}$ metabolite) did not alter alveolarization or postnatal lung function in preterm sheep [21]. Furthermore, in term-born rats, retinoic acid administration to newborn pups did not attenuate stunted alveolarization provoked by oxygen toxicity [22], but did promote increased alveolar growth during a post-hyperoxia room air recovery period [23]. As such, studies on vitamin A in experimental animal models of BPD provided very limited support at the preclinical level to the successful clinical trials. However, experimental animal models of BPD have subsequently provided a means of exploring the utility of combinations of different vitamin A metabolites to promote postnatal lung growth and development [24]. Beyond the lung, while the administration of vitamin A metabolites to hyperoxia-exposed newborn mice attenuated later neurobehavioral impairments [25], a 2016 systematic review suggested no evidence of either benefit or harm to the longterm neurodevelopmental status from vitamin A supplementation in very-low-birth-weight infants [26]. Thus, a disparity exists between outcomes of vitamin A interventions in preclinical and those in clinical studies on BPD. Different vitamin A dosing regimens, or speciesdependent responses either to vitamin A metabolites or to the injurious stimuli employed (such as oxygen toxicity), have been proposed to underlie the lack of effect of vitamin A on lung maturation in experimental animal models of BPD [21].

Similar to studies on vitamin A, preclinical studies on the utility of caffeine and other methylxanthines to manage BPD largely came after the demonstrated success of the intervention in clinical trials. Caffeine, first used to manage apnea of prematurity in the 1970s [reviewed in 27 ] in infants with or at risk for BPD, has been supported by multiple RCTs, starting in 2006 [28, 29]. However, studies on caffeine in experimental animal models came later, and have yielded mixed and often conflicting results [30], where caffeine administration was either protective [31] or without effect [32] or exacerbated stunting of lung development provoked by oxygen toxicity in term mouse models of BPD [33]. These diverse effects were attributed to different oxygen toxicity protocols, different mouse strains and different caffeine dosing [30]. In contrast, in animal species other than mice - that is, in term rats [34]

Identifying Pathogenic Pathways and Disease Management Targets in BPD and preterm rabbits [35] - caffeine administration was consistently protective against the deleterious effect of oxygen toxicity on lung development. Thus, while mousebased experimental animal models of BPD did not highlight caffeine as a candidate therapeutic intervention to be tested in clinical trials, a combination of studies using other animal models appears to support the testing of potentially useful pharmacological interventions to manage BPD.

From 1996 to 2005, 10 RCTs examined the ability of dexamethasone administration to prevent BPD [36], with the first BPD dexamethasone RCT having taken place in 1983 [37]. While administration of dexamethasone is effective at preventing BPD, this benefit does not outweigh the risk of long-term adverse neurological outcomes [19]. However, there may still be a place for low-dose dexamethasone treatment of infants with BPD who are ventilator dependent after the first week of life [36]. In contrast to the studies reported above for vitamin A and caffeine, some preclinical studies do predate the first dexamethasone BPD trial. These preclinical studies include the demonstration that postnatal dexamethasone partially rescued lung growth under conditions of caloric restriction in newborn guinea pigs [38]. As such, these and other preclinical studies may have contributed to the enthusiasm for the evaluation of dexamethasone in the management of preterm birth. However, postnatal administration of dexamethasone failed to reduce oxygen-induced lung injury in preterm guinea pigs [39], indicating the existence of preclinical studies that also spoke against the use of dexamethasone in preterm infants.

The last 2 of the 5 recent pharmacological interventions validated in RCTs - inositol and clarithromycin were tested in single-center RCTs, and their efficacy has not been confirmed $[19,40]$. Inositol has not received any attention in experimental animal models of BPD. Clarithromycin administered alone was documented to be ineffective in limiting the deleterious effect of oxygen toxicity on postnatal lung maturation in rats [41], although clarithromycin administered together with montelukast and pentoxifylline showed promise. Thus, neither inositol nor clarithromycin was identified as a candidate therapeutic intervention in preclinical models of BPD.

The rather dismal success of 5 out of 21 recent pharmacological interventions has raised questions about the low success rate of the drugs employed in these RCTs. Better trial designs including considerations about pharmacokinetics and appropriate endpoints, the need for multicenter collaboration, and problems related to the clinical definition of BPD [40] have been identified as po- 
tential reasons why some of the pharmacological interventions may have failed to prevent BPD. Interestingly, none of these 5 "successful" pharmacological interventions was first identified in experimental animal models of BPD.

A search of the ClinicalTrials.gov database (accessed on February 10, 2020) using the search term "bronchopulmonary dysplasia" yielded 219 registered studies, of which 16 address cell therapy and 67 evaluate various aspects of pharmacological interventions. These interventions include a number of compounds that have been identified as being protective in preclinical studies undertaken in experimental animal models of BPD, including L-citrulline [42], $\omega-3$ fatty acids [43], sildenafil [44] and vitamin D [45]. Additionally, some pharmacological agents now being evaluated in clinical trials have not demonstrated any utility in preclinical studies, such as montelukast, which when applied alone did not protect against hyperoxia-induced stunting of alveolarization in newborn rats [46]. The outcomes of these ongoing trials are eagerly awaited, and they will reveal whether preclinical studies in experimental animal models of BPD proved useful for the identification of further avenues for the management of BPD. Should that indeed be the case, a plethora of other candidate targets exists, which may suggest additional candidates for further assessment. Over the past 4 years alone, 212 pharmacological interventions and 157 genetic interference studies have been undertaken in experimental animals and in experimental animal models of BPD [reviewed in 5,47], in order to identify mediators of normal and stunted lung development, as well as to promote improved lung maturation under pathological conditions. The next generation of clinically applicable pharmacological interventions may well be amongst those mediators of normal and aberrant lung development that have been identified in preclinical studies in experimental animal models of BPD. Particularly exciting candidates amongst these include growth factor signaling, such as transforming growth factor- $\beta$, as well as mediators of inflammation, including the antagonism of interleukin-1 receptor-mediated signaling and of course the spectrum of cell-based therapies that are currently emerging.

No report on the utility of preclinical studies would be complete without some critique of the experimental animal models currently in widespread use. It is evident from the discussion above, and other reviews addressing this topic [48], that the seminal discoveries highlighting the utility of surfactant and antenatal corticosteroids were made in preterm rabbit, sheep and primate models. The selection of experimental animal models in preclinical studies on BPD has shifted over time. Where the preterm primate, rabbit and lamb models were formerly the experimental animal models of choice, for logistical, ethical and financial reasons, these models are used less frequently today. Many nations now forbid the use of nonhuman primates in preclinical studies, and there are substantial labor and other costs associated with preterm lamb models. These models, as well as the preterm rabbit model, have largely been replaced by the term-born mouse and rat models. There are several reasons for this, including that (1) the costs of the animals, as well as the animal husbandry, are considerably cheaper for mice and rats; (2) due to the comparatively small size of mouse and rat pups, a reduced amount of the interventional agents is required to be administered to the pups, as compared to the amount that would be used, for example, for much larger preterm lambs; (3) the shorter period of postnatal lung maturation in rodents, compared to primates and lambs, means that experimental time courses are shorter and results can be obtained faster and at lower cost; and (4) particularly in the case of mice, extensive genetic tools are available, including comprehensive knowledge of genomes, and widespread availability of transgenic mice.

However, this move in the direction of rodent models, particularly mouse models, has multiple important drawbacks. The most notable drawback for drug discovery studies is the lack of the element of prematurity in rodent models. While it is true that mouse and rat pups are born in the saccular stage of lung development, the same stage in which many preterm infants are born that go on to develop BPD, these "saccular" mouse and rat pups are fully competent for gas exchange and have no medical need for any kind of oxygen supplementation. This contrasts sharply with the clinical situation, where the very definition of BPD rests on the need for supplemental oxygen [49]. A second drawback of mouse models, in particular, is that mice cluster together separately from all other species in preclinical studies on pharmacological interventions in experimental animal models of BPD. The studies on the utility of caffeine administration to promote lung maturation described above are a case in point. A recent overview of preclinical studies on caffeine and other methylxanthines [30] reported that caffeine (or other methylxanthines) exhibited a positive effect on lung maturation or respiratory function in rats, rabbits, lambs and baboons, while in mice, caffeine either exhibited no effect at all or had a deleterious effect on lung maturation. As such, mice appear to behave differently (indeed oppositely) to all other species in terms of responses to candidate 
therapies for BPD. This raises an important point of caution and highlights the need to validate any interventional study in a species other than the mouse prior to proceeding further with a candidate intervention. These concerns highlight a pressing need to promote the widespread use of experimental animals other than the laboratory mouse - in particular, experimental animals that can be delivered preterm - in an effort to mimic the clinical scenario as well as possible.

Another point of consideration is the selection of appropriate endpoints in preclinical studies, particularly those studies employing rats or mice. Most preclinical studies in rats or mice focus heavily on the alveolar simplification aspect of "new" BPD. That is, almost every study in rats or mice measures the lung structure (primarily, the size or number of alveoli; directly or appropriate surrogates). However, these studies focus overtly on only one particular element of BPD pathophysiology: lung alveolarization. This is problematic for several reasons. Firstly, other key pathophysiological hallmarks of BPD are largely ignored. Remaining within the cardiopulmonary system, there is little consideration of breathing or respiratory function. This is largely due to difficulty with performing breathing mechanics studies on immature rodents, due to their small size. The signal-to-noise ratio complicates the derivation of inspiration/expiration loops from newborn mice studies using whole-body plethysmography. Furthermore, the small internal diameter of the trachea of newborn mice and mouse pups creates technical difficulties with intubation and airway cannula resistance in forced oscillation studies, for example, using the flexiVent approach. However, efforts to address this important deficit in our knowledge and technology are already bearing fruit, with the first report on the impact of hyperoxia exposure in the early postnatal period in rats in the control of breathing [50]. Secondly, the small size of newborn mice in particular makes any assessment of pulmonary hemodynamics impossible. Right-heart catheterization is technically impossible, and the small size of the experimental animals also makes both echocardiography and computed axial tomography approaches problematic. Thirdly, again related to the small size of newborn mice, determination of any diffusion coefficients - for example, for carbon monoxide - is impossible. Thus, while it is possible to acquire excellent information about the lung structure, the increasingly widespread use of rodents as experimental animals in preclinical studies on BPD has also resulted in limited knowledge about the effect of pharmacological and genetic intervention on breathing mechanics and function, as well as pul-

Identifying Pathogenic Pathways and Disease Management Targets in BPD monary hemodynamics. This is seen as an important limitation of rodent-based experimental animal models of BPD.

The nature of the injurious stimulus employed in experimental animal models of BPD also warrants discussion. In a clinical setting, it is desirable to use as low an $\mathrm{FiO}_{2}$ as possible. However, hyperoxia-based studies with experimental animals continue to routinely employ an $\mathrm{FiO}_{2}$ of $0.80-1.00$ [51]. This is largely in an effort to mimic structural changes that are noted in autopsy material from BPD nonsurvivors. Two key issues emerge here. Firstly, most autopsy material from BPD nonsurvivors available to date is derived from patients with "old" BPD, and it is highly desirable to reassess what perturbations to the lung architecture exist in the lungs of patients with "new" BPD. Once this is known, injurious stimuli can be developed and refined, in order to properly mimic those perturbations to the lung structure seen in infants with BPD in the lungs of experimental animals in which BPD is being modeled. Secondly, the injurious stimuli employed to provoke lung injury in experimental animal models must better reflect the injurious stimuli in affected BPD patients. It is evident that the $\mathrm{FiO}_{2}$ levels currently employed in experimental studies are not comparable with those used clinically. Additionally, there is a need for development of more complex and representative injurious stimuli. BPD patients have immature lungs that are injured by a combination of oxygen toxicity, infection, and other factors. Thus, the injury stimulus is multifactorial. This is not the case in most rodent models, where hyperoxia alone is frequently employed. There is a clear need for development of more complex injurious stimuli that encompass the spectrum of prematurity, oxygen toxicity, and infection.

In summary, experimental animal models have been extremely valuable in the development of hallmark clinical management strategies for BPD, including surfactant replacement and antenatal corticosteroids. While a move away from large animal models of BPD in favor of termborn rodents has facilitated the identification of a multitude of new mechanisms of normal and stunted lung development, this has come at a cost: (1) the loss of information about breathing mechanics, gas exchange, and pulmonary hemodynamics; and (2) a less relevant clinical context, where lung prematurity and a background of infection are now rarely present in the pathophysiology under study. There is a pressing need to refine existing models to better recapitulate pathological processes at play in affected infants, in order to better evaluate new candidate pharmacological interventions for the management of BPD. 


\section{Disclosure Statement}

The author has no conflicts of interest to declare.

\section{Funding Sources}

The author was supported by the Max Planck Society; Rhön Klinikum AG grant FI_66; University Hospital Giessen and Marburg grant UKGM62589135; the Federal Ministry of Higher Edu- cation, Research and the Arts of the State of Hesse "LOEWE Programme"; the German Center for Lung Research (Deutsches Zentrum für Lungenforschung); and the German Research Foundation (Deutsche Forschungsgemeinschaft) through Excellence Cluster EXC147, Collaborative Research Center SFB1213/1, Clinical Research Unit KFO309/1, and individual research grant Mo $1789 / 1$.

\section{References}

1 Northway WH Jr, Rosan RC, Porter DY. Pulmonary disease following respirator therapy of hyaline-membrane disease. Bronchopulmonary dysplasia. N Engl J Med. 1967 Feb; 276(7):357-68

2 Baraldi E, Filippone M. Chronic lung disease after premature birth. N Engl J Med. 2007 Nov;357(19): 1946-55.

3 Stoll BJ, Hansen NI, Bell EF, Shankaran S, Laptook AR, Walsh MC, et al.; Eunice Kennedy Shriver National Institute of Child Health and Human Development Neonatal Research Network. Neonatal outcomes of extremely preterm infants from the NICHD Neonatal Research Network. Pediatrics. 2010 Sep;126(3):443-56.

4 Jobe AH. The new bronchopulmonary dysplasia. Curr Opin Pediatr. 2011 Apr;23(2): $167-72$.

5 Lignelli E, Palumbo F, Myti D, Morty RE. Recent advances in our understanding of the mechanisms of lung alveolarization and bronchopulmonary dysplasia. Am J Physiol Lung Cell Mol Physiol.2019Dec;317(6):L83287.

6 Northway WH Jr, Rosan RC, Shahinian L Jr, Castellino RA, Gyepes MT, Durbridge T. Radiologic and histologic investigation of pulmonary oxygen toxicity in newborn guinea pigs. Invest Radiol. 1969 May-Jun;4(3):14855.

7 Bonikos DS, Bensch KG, Ludwin SK, Northway WH Jr. Oxygen toxicity in the newborn. The effect of prolonged 100 per cent $\mathrm{O} 2$ exposure on the lungs of newborn mice. Lab Invest. 1975 May;32(5):619-35.

8 Ambalavanan N, Morty RE. Searching for better animal models of BPD: a perspective. Am J Physiol Lung Cell Mol Physiol. 2016 Nov;311(5):L924-7.

9 Nardiello C, Mižíková I, Morty RE. Looking ahead: where to next for animal models of bronchopulmonary dysplasia? Cell Tissue Res. 2017 Mar;367(3):457-68.

10 Jobe AH. Pulmonary surfactant therapy. N Engl J Med. 1993 Mar;328(12):861-8.

11 Speer CP, Sweet DG, Halliday HL. Surfactant therapy: past, present and future. Early Hum Dev. 2013 Jun;89 Suppl 1:S22-4.

12 Roberts D, Brown J, Medley N, Dalziel SR. Antenatal corticosteroids for accelerating fe- tal lung maturation for women at risk of preterm birth. Cochrane Database Syst Rev. 2017 Mar;3:CD004454.

13 Avery ME, Mead J. Surface properties in relation to atelectasis and hyaline membrane disease. AMA J Dis Child. 1959 May;97(5, Part 1):517-23.

14 Fujiwara T, Maeta H, Chida S, Morita T, Watabe Y, Abe T. Artificial surfactant therapy in hyaline-membrane disease. Lancet. 1980 Jan; 1(8159):55-9.

15 Liggins GC. Premature delivery of foetal lambs infused with glucocorticoids. J Endocrinol. 1969 Dec;45(4):515-23.

16 Liggins GC, Howie RN. A controlled trial of antepartum glucocorticoid treatment for prevention of the respiratory distress syndrome in premature infants. Pediatrics. 1972 Oct; 50(4):515-25.

17 Taeusch HW Jr, Heitner M, Avery ME. Accelerated lung maturation and increased survival in premature rabbits treated with hydrocortisone. Am Rev Respir Dis. 1972 Jun; 105(6):971-3

18 Frank L, Summerville J, Massaro D. The effect of prenatal dexamethasone treatment on oxygen toxicity in the newborn rat. Pediatrics. 1980 Feb;65(2):287-93.

19 Beam KS, Aliaga S, Ahlfeld SK, CohenWolkowiez M, Smith PB, Laughon MM. A systematic review of randomized controlled trials for the prevention of bronchopulmonary dysplasia in infants. J Perinatol. 2014 Sep; 34(9):705-10.

20 Ong DE, Chytil F. Changes in levels of cellular retinol- and retinoic-acid-binding proteins of liver and lung during perinatal development of rat. Proc Natl Acad Sci USA. 1976 Nov; 73(11):3976-8.

21 Willet KE, Jobe AH, Ikegami M, Newnham J, Sly PD. Antenatal retinoic acid does not alter alveolization or postnatal lung function in preterm sheep. Eur Respir J. 2000 Jul;16(1): 101-7.

22 Veness-Meehan KA, Bottone FG Jr, Stiles AD. Effects of retinoic acid on airspace development and lung collagen in hyperoxia-exposed newborn rats. Pediatr Res. 2000 Oct;48(4): 434-44.

23 Veness-Meehan KA, Pierce RA, Moats-Staats BM, Stiles AD. Retinoic acid attenuates O2- induced inhibition of lung septation. Am J Physiol Lung Cell Mol Physiol. 2002 Nov; 283(5):L971-80.

24 James ML, Ross AC, Nicola T, Steele C, Ambalavanan N. VARA attenuates hyperoxiainduced impaired alveolar development and lung function in newborn mice. Am J Physiol Lung Cell Mol Physiol. 2013 Jun;304(11): L803-12.

25 Ramani M, van Groen T, Kadish I, Ambalavanan N, McMahon LL. Vitamin A and retinoic acid combination attenuates neonatal hyperoxia-induced neurobehavioral impairment in adult mice. Neurobiol Learn Mem. 2017 May; 141:209-16.

26 Darlow BA, Graham PJ, Rojas-Reyes MX. Vitamin A supplementation to prevent mortality and short- and long-term morbidity in very low birth weight infants. Cochrane Database Syst Rev. 2016 Aug;(8): CD000501

27 Kreutzer K, Bassler D. Caffeine for apnea of prematurity: a neonatal success story. Neonatology. 2014;105(4):332-6.

28 Schmidt B, Roberts RS, Davis P, Doyle LW, Barrington KJ, Ohlsson A, et al.; Caffeine for Apnea of Prematurity Trial Group. Caffeine therapy for apnea of prematurity. N Engl J Med. 2006 May;354(20):2112-21.

29 Schmidt B, Roberts RS, Davis P, Doyle LW, Barrington KJ, Ohlsson A, et al. Long-term effects of caffeine therapy for apnea of prematurity. N Engl J Med. 2007 Nov;357(19):1893902.

30 Rath P, Nardiello C, Morty RE. A new target for caffeine in the developing lung: endoplasmic reticulum stress? Am J Physiol Lung Cell Mol Physiol. 2017 Oct;313(4): L659-63.

31 Dumpa V, Nielsen L, Wang H, Kumar VH. Caffeine is associated with improved alveolarization and angiogenesis in male mice following hyperoxia induced lung injury. BMC Pulm Med. 2019 Jul;19(1):138

32 Rath P, Nardiello C, Surate Solaligue DE, Agius R, Mižíková I, Hühn S, et al. Caffeine administration modulates TGF- $\beta$ signaling but does not attenuate blunted alveolarization in a hyperoxia-based mouse model of bronchopulmonary dysplasia. Pediatr Res. 2017 May; 81(5):795-805. 
33 Dayanim S, Lopez B, Maisonet TM, Grewal S, Londhe VA. Caffeine induces alveolar apoptosis in the hyperoxia-exposed developing mouse lung. Pediatr Res. 2014 Mar;75(3): 395-402.

34 Teng RJ, Jing X, Michalkiewicz T, Afolayan AJ, Wu TJ, Konduri GG. Attenuation of endoplasmic reticulum stress by caffeine ameliorates hyperoxia-induced lung injury. Am J Physiol Lung Cell Mol Physiol. 2017 May; 312(5):L586-98.

35 Nagatomo T, Jiménez J, Richter J, De Baere S, Vanoirbeek J, Naulaers G, et al. Caffeine prevents hyperoxia-induced functional and structural lung damage in preterm rabbits. Neonatology. 2016;109(4):274-81.

36 Halliday HL. Update on postnatal steroids. Neonatology. 2017;111(4):415-22.

37 Mammel MC, Green TP, Johnson DE, Thompson TR. Controlled trial of dexamethasone therapy in infants with bronchopulmonary dysplasia. Lancet. 1983 Jun;1(8338): 1356-8.

38 Lechner AJ. Interaction of prenatal starvation and dexamethasone treatment on lung development in newborn guinea pigs. Am Rev Respir Dis. 1987 May;135(5):991-6.

39 Town GI, Phillips GJ, Landreau M, Louden J, Holgate ST, Kelly FJ. Dexamethasone treatment fails to reduce oxygen-induced lung injury in the preterm guinea pig. Effects on pulmonary inflammation and antioxidant status. Biochem Pharmacol. 1993 Nov;46(9):156572.
40 Viscardi RM. Pharmacological options for BPD prevention: steps for better clinical trial design. J Perinatol. 2014 Sep;34(9):656-7.

41 Demir K, Kumral A, Duman N, Sarioglu S, Yilmaz O, Yesilirmak DC, et al. Clarithromycin, montelukast, and pentoxifylline combination treatment ameliorates experimental neonatal hyperoxic lung injury. J Matern Fetal Neonatal Med. 2008 Jun;21(6):407-13.

42 Vadivel A, Aschner JL, Rey-Parra GJ, Magarik J, Zeng H, Summar M, et al. L-citrulline attenuates arrested alveolar growth and pulmonary hypertension in oxygen-induced lung injury in newborn rats. Pediatr Res. 2010 Dec; 68(6):519-25

43 Velten M, Britt RD Jr, Heyob KM, Tipple TE, Rogers LK. Maternal dietary docosahexaenoic acid supplementation attenuates fetal growth restriction and enhances pulmonary function in a newborn mouse model of perinatal inflammation. J Nutr. 2014 Mar;144(3): 258-66.

44 Ladha F, Bonnet S, Eaton F, Hashimoto K, Korbutt G, Thébaud B. Sildenafil improves alveolar growth and pulmonary hypertension in hyperoxia-induced lung injury. Am J Respir Crit Care Med. 2005 Sep;172(6):750-6.

45 Kose M, Bastug O, Sonmez MF, Per S, Ozdemir A, Kaymak E, et al. Protective effect of vitamin $D$ against hyperoxia-induced lung injury in newborn rats. Pediatr Pulmonol. 2017 Jan;52(1):69-76.
46 Jouvencel P, Fayon M, Choukroun ML, Carles D, Montaudon D, Dumas E, et al. Montelukast does not protect against hyperoxia-induced inhibition of alveolarization in newborn rats. Pediatr Pulmonol. 2003 Jun;35(6): 446-51.

47 Surate Solaligue DE, Rodríguez-Castillo JA, Ahlbrecht K, Morty RE. Recent advances in our understanding of the mechanisms of late lung development and bronchopulmonary dysplasia. Am J Physiol Lung Cell Mol Physiol. 2017 Dec;313(6):L1101-53.

48 Jobe AH. Animal models, learning lessons to prevent and treat neonatal chronic lung disease. Front Med (Lausanne). 2015 Aug;2: 49.

49 Ibrahim J, Bhandari V. The definition of bronchopulmonary dysplasia: an evolving dilemma. Pediatr Res. 2018 Nov;84(5):586-8.

50 Mouradian GC Jr, Alvarez-Argote S, Gorzek R, Thuku G, Michkalkiewicz T, Wong-Riley MT, et al. Acute and chronic changes in the control of breathing in a rat model of bronchopulmonary dysplasia. Am J Physiol Lung Cell Mol Physiol. 2019 Mar;316 (3):L506-18.

51 Silva DM, Nardiello C, Pozarska A, Morty RE. Recent advances in the mechanisms of lung alveolarization and the pathogenesis of bronchopulmonary dysplasia. Am J Physiol Lung Cell Mol Physiol. 2015 Dec;309(11):L123972. 